\title{
National burden of colorectal cancer in Lithuania and the ranking of Lithuania within the 45 European nations
}

\author{
RAIMUNDAS LUNEVICIUS ${ }^{1,2}$, TOMAS POSKUS ${ }^{2}$ and NARIMANTAS E. SAMALAVICIUS ${ }^{3}$
}

\author{
${ }^{1}$ Emergency General Surgery and Major Trauma Centre, Department of General Surgery, \\ Aintree University Hospital NHS Foundation Trust, School of Medicine, University of Liverpool, Liverpool, \\ Merseyside L9 7AL, UK; ${ }^{2}$ Centre of Abdominal Surgery, Clinic of Gastroenterology, Nephrourology and Surgery, \\ Faculty of Medicine, Vilnius University, Vilnius LT-08661; ${ }^{3}$ Clinic of Internal Diseases, Family Medicine and Oncology, \\ Faculty of Medicine, National Cancer Institute, Vilnius University, Vilnius LT-08660, Lithuania
}

Received June 30, 2014; Accepted March 17, 2015

DOI: $10.3892 / 01.2015 .3171$

\begin{abstract}
The aim of the present paper was to assess the national burden of colorectal cancer in Lithuania, and to determine the performance of Lithuania for the control of colorectal cancer compared with 45 European nations by ranking. Seven sources of data and information were used. The majority of the findings on disease burden are reported in the present study in the form of the crude and age-standardised incidence, age-specific and age-standardised mortality, disability adjusted life years (DALYs), years lived with disability (YLDs) and years of life lost (YLLs) per 100,000 individuals per year by gender and in the two genders combined between 1990 and 2011. Colorectal cancer was ranked as the 3rd leading cause of disease burden out of all malignancies. Overall, the crude incidence was 35 per 100,000 individuals in 2001 and 51 per 100,000 individuals in 2011 in Lithuania. Incidence and mortality from colorectal cancer varied markedly within Lithuania. The number of DALYs and YLLs slightly declined between 2005 and 2010. YLLs contributed $97 \%$ of the total burden due to colorectal cancer in 1990, and $96 \%$ in 2010 . The mortality rate was 4-5 times higher in males aged 50-54 years than in males aged 45-49. The YLDs per 100,000 individuals per year in Lithuania increased by $61.1 \%$ between 1990 and 2010. However, Lithuania was below the average of the Central $\&$ Eastern, Southern, Northern and Western regions of Europe.
\end{abstract}

Correspondence to: Mr. Raimundas Lunevicius, Department of General Surgery, Aintree University Hospital NHS Foundation Trust, School of Medicine, University of Liverpool, Lower Lane, Liverpool, Merseyside L9 7AL, UK

E-mail: raimundas.lunevicius@aintree.nhs.uk

Abbreviations: GBD, global burden of disease; DALYs, disability-adjusted life years; YLLs, years of life lost; YLDs, years lived with a disability; NBD, national burden of disease

Key words: colorectal cancer, incidence, mortality, burden of disease, disability-adjusted life years, Lithuania
It was concluded that the national colorectal cancer service provision should be amplified and that a programme for the prevention and control of colorectal cancer is required.

\section{Introduction}

The concept of global burden of disease (GBD) emerged when it was perceived that the use of mortality as a measure of disease burden reflects only a section of population health and this metric does not incorporate the impact of disability (1-4). A key measure for GBD is the number of disability adjusted life years (DALYs), which is calculated from the sum of two other measures, consisting of the years of life lost (YLLs) and years lived with disability (YLDs) (5). These composite measures incorporate and synthesize estimates of the incidence, prevalence and duration of a particular condition, and the outcome, either disability or premature death. The measures were developed for the objective assessment of the burden of disease within the population of one country, at a national or subnational level, and within populations of regions and continents $(2,6,7)$.

Four factors determined the conception and design of this study. The first was that malignancies have not been previously ranked by national burden of disease (NBD) in Lithuania with reference to the composite single indicators. The second was that there is no data on the national burden of colorectal cancer in the population of Lithuania at present. The third reason was that there is no comparative assessment between the performance of Lithuania for colorectal cancer and other European nations based on ranking. The fourth was that objective assessment of NBD is required prior to the complete implementation of a national colorectal cancer screening and prevention programme in Lithuania.

The analysis that follows, therefore, elaborates on the national burden of all malignancies and colorectal cancer in Lithuania. The performance of Lithuania in terms of colorectal cancer is assessed by comparison with 45 European nations by their ranking. The present study aimed to produce trends for the incidence of colorectal cancer, and also for the mortality, DALYs, YLLs and YLDs due to colorectal cancer. The percentage change of the aforementioned measures was 
assessed for countries and regions between 1990 and 2010. The ranking of Lithuania was also assessed to determine whether the country was above or below the average of the currently named or historic European regions. An additional reason for the production of the present study was to encourage the adoption of the composite metrics of GBD as a health policy so that estimates of NBD may be produced for Lithuania and its regions on a regular basis (8).

\section{Materials and methods}

Seven sources of data and information were used for the creation and analysis of the present figures and tables. These sources were the WHO Department of Measurement and Health Information (8), International Agency for Research on Cancer, GLOBOCAN (9), Lithuanian Health Information Centre (10), WHO European Detailed Mortality Database (11), Global Burden of Disease Study, Institute for Health Metrics and Evaluation (12), Statistics Lithuania (13) and Lithuanian Cancer Registry (14). The majority of the data on disease burden are reported in the present study by gender as age-standardised DALYs, YLLs and YLDs per 100,000 individuals per year. The WHO population age standard for each gender was used for the expression of age-standardised rates $(8,9,11,12)$. Other metrics, including the crude incidence rate and mortality, age-standardised and age-specific mortality rates for colorectal cancer were also used in the present study for the clarification of anomalies in the NBD of Lithuania. The International Statistical Classification of Diseases and Related Health Problems (10th revision), codes C18-21 for malignant neoplasms of the colon, rectosigmoid junction, rectum, anal canal and anus (http://apps.who.int/classifications/icd10/browse/2015/en) were used to search for incidence and mortality data within the Health Indicators of the Lithuania database and the European Detailed Mortality Database $(10,11)$.

The comparative analysis of the burden of colorectal cancer incorporated the data from 45 European nations. The present study aimed to produce trends for the incidence and mortality of colorectal cancer, and the DALYs, YLLs and YLDs due to this disease. The percentage change of these measures in countries and regions between 1990 and 2000 was also determined.

The data from Lithuania were assessed to determine if the country was above or below the average of the currently named or historic European regions. The Northern, Southern, Western, and Central and Eastern regions of Europe were defined by GLOBOCAN 2008 (9). The original EU-15 consisted of the following countries: Denmark, Germany, the UK, Belgium, Luxembourg, Austria, Italy, Netherlands, Ireland, France, Sweden, Portugal, Spain, Finland and Greece. The terms 'communist countries in 1990' and 'democratic countries in 1990' are historic. The first term includes all the communist countries of Europe in 1990, with the exception of the German Democratic Republic.

\section{Results}

Leading causes of NBD. The fourth leading cause of NBD is malignancies, with the preceding three causes being injuries, neuropsychiatric conditions and cardiovascular conditions in Lithuania. Malignancies account for $9.8 \%$ of the disease burden resulting from males, with 2098 DALYs per 100,000 males in 2004 , and $11.2 \%$ of the disease burden that arises from females, with 1369 DALYs per 100,000 females in 2004. Malignancies are the fourth leading cause of the NBD accounted for by males, preceded by injuries (26.6\%), cardiovascular diseases $(21.1 \%)$ and neuropsychiatric conditions (18\%). In females, malignancies rank third, being preceded by neuropsychiatric conditions $(25.7 \%)$ and cardiovascular diseases $(15.2 \%)$.

Ranking of malignancies by DALYs and YLDs. The national burden of 10 malignant oncological diseases is reported and ranked in Table I. Colorectal cancer was the third (8.6\%) leading cause of DALYs in males in Lithuania in 2008, preceded by lung $(23.8 \%)$ and stomach $(9.9 \%)$ cancers. In the female population, colorectal cancer was also the third leading cause of DALYs, preceded by breast (21.3\%) and uterine cervix $(11.8 \%)$ cancers. The burden of malignant disease was $37.3 \%$ higher in males than females in Lithuania in 2008 .

Table I also reveals the contribution of the YLDs accounted for by the 10 leading malignant diseases to the total disability-associated burden of malignant diseases for Lithuania. The disability burden of malignant disease was revealed to be $14.4 \%$ higher in females than males. Prostate and colorectal cancers were the leading contributors, accounting for almost $45.8 \%$ of the disease burden due to disability in the male population. In females, colorectal cancer was the fourth leading cause $(9.4 \%)$, preceded by breast $(39.3 \%)$ and uterine cancers of either the cervix or corpus $(28 \%)$.

Incidence of colorectal cancer and the ranking of Lithuania. Overall, the crude incidence of colorectal cancer in Lithuania per 100,000 individuals was 35 in 2001 and 51 in 2011. The overall incidence varied between the ten counties and five cities of Lithuania. In 2011, the incidence was 68 and 42 cases per 100,000 individuals in the cities Vilnius and Kaunas, and 62 and 35 cases per 100,000 individuals in the counties Panevėžys and Tauragè. The incidence of colorectal cancer increased in all Lithuanian regions, with the exception of Western Lithuania. The incidence decreased in Western Lithuania over the 11 years. The overall age-standardised incidence of colorectal cancer for the two genders was 25 cases per 100,000 individuals in Lithuania, resulting in Lithuania being ranked as the 27 th country out of the comparative nations in 2008. The overall age-standardised incidence of colorectal cancer for the two genders was highest in the Central European countries of Slovakia, Hungary and the Czech Republic in 2008, with 42, 41 and 39 cases per 100,000 individuals, respectively.

With the age-standardised incidence of 33 colorectal cancer cases per 100,000 males, Lithuania was ranked 22nd out of the 45 European countries. The same incidence was estimated in Poland. The incidence of colorectal cancer in males was highest in Slovakia, Hungary and the Czech Republic, with 61, 56 and 54 cases per 100,000 males, respectively. In females, the incidence of colorectal cancer was highest in Israel, Norway, and Denmark, with 36, 34 and 34 cases per 100,000 females, respectively. Lithuania, 
Table I. Ten causes of the national burden of malignancies ranked by DALYs and YLDs in 100,000 men and 100,000 women in 2008, Lithuania.

A, Male patients $(n=100,000)$

\begin{tabular}{|c|c|c|c|c|c|c|}
\hline \multirow[b]{2}{*}{ Rank } & \multicolumn{3}{|c|}{ DALYs } & \multicolumn{3}{|c|}{ YLDs } \\
\hline & Cancer & $\mathrm{n}$ & $\%$ & Cancer & $\mathrm{n}$ & $\%$ \\
\hline 1 & Lung & 886 & 23.8 & Prostate & 78 & 28.1 \\
\hline 2 & Stomach & 368 & 9.9 & Colorectum & 48 & 17.3 \\
\hline 3 & Colorectum & 321 & 8.6 & Lung & 28 & 10.1 \\
\hline 4 & Prostate & 306 & 8.2 & Stomach & 21 & 7.6 \\
\hline 5 & Leukaemia & 182 & 4.9 & Bladder & 14 & 5.0 \\
\hline 6 & Pancreas & 177 & 4.8 & Lip, oral cavity & 12 & 4.3 \\
\hline 7 & Kidney & 152 & 4.1 & Kidney & 10 & 3.6 \\
\hline 8 & Oesophagus & 152 & 4.1 & Other pharynx & 10 & 3.6 \\
\hline 9 & Nervous system & 152 & 4.1 & Larynx & 9 & 3.2 \\
\hline \multirow[t]{2}{*}{10} & Larynx & 135 & 3.6 & Non-Hodgkin's lymphoma & 6 & 2.2 \\
\hline & All cancers ${ }^{\mathrm{a}}$ & 3721 & 100.0 & All cancers ${ }^{\mathrm{a}}$ & 278 & 100.0 \\
\hline
\end{tabular}

B, Female patients $(n=100,000)$

\begin{tabular}{|c|c|c|c|c|c|c|}
\hline \multirow[b]{2}{*}{ Rank } & \multicolumn{3}{|c|}{ DALYs } & \multicolumn{3}{|c|}{ YLDs } \\
\hline & Cancer & $\mathrm{n}$ & $\%$ & Cancer & $\mathrm{n}$ & $\%$ \\
\hline 1 & Breast & 577 & 21.3 & Breast & 125 & 39.3 \\
\hline 2 & Uterine cervix & 319 & 11.8 & Uterine cervix & 52 & 16.4 \\
\hline 3 & Colorectum & 240 & 8.9 & Uterine corpus & 37 & 11.6 \\
\hline 4 & Ovary & 208 & 7.7 & Colorectum & 30 & 9.4 \\
\hline 5 & Stomach & 190 & 7.0 & Ovary & 19 & 6.0 \\
\hline 6 & Brain, nervous system & 151 & 5.6 & Stomach & 9 & 2.8 \\
\hline 7 & Lung & 128 & 4.7 & Non-Hodgkin's lymphoma & 5 & 1.6 \\
\hline 8 & Leukaemia & 118 & 4.4 & Kidney & 5 & 1.6 \\
\hline 9 & Uterine corpus & 99 & 3.7 & Melanoma of skin & 4 & 1.3 \\
\hline \multirow[t]{2}{*}{10} & Pancreas & 96 & 3.5 & Thyroid & 4 & 1.3 \\
\hline & All cancers ${ }^{\mathrm{a}}$ & 2711 & 100.0 & All cancers ${ }^{\mathrm{a}}$ & 318 & 100.0 \\
\hline
\end{tabular}

DALYs, disability-adjusted life years; YLDs, years lived with disability; \%, percentage out of total burden caused by all cancers in men or in women. ${ }^{a}$ All cancers excluding non-melanoma skin cancer (Ferlay et al) (9).

with the age-standardised incidence rate of 20 cases per 100,000 females, was ranked as 29th in 2008, the same ranking as the Republic of Moldova, Austria and Latvia.

Mortality due to colorectal cancer. There were 719 mortalities associated with colorectal cancer in Lithuania in 1990, out of a total population of 3.67 million individuals (12). Despite the decrease in population size of $16 \%$ in 2010,958 mortalities were registered as associated with colorectal cancer in 2010 , resulting in a $24.9 \%$ increase in colorectal cancer-associated mortalities between 1990 and 2010. This resulted in a sharp $36.7 \%$ increase in the male mortality rate, $27.6 \%$ increase in the female mortality rate, and $32.1 \%$ increase in the overall mortality rate associated with colorectal cancer in the two genders between 1990 and 2010. It is important to note that the trends based on different sources of information vary slightly $(11,12)$, as demonstrated by Fig. 1 .

Mortality pattern by age and gender. Fig. 2 depicts a pattern based on the crude age-specific mortality rates of colorectal cancer by age, gender and the two genders combined in 1998 and 2010 in Lithuania. All six mortality curves in the Fig. 2 began to increase in the age group of 45-49 years. The mortality rate was 4-5 times higher in males aged 50-54 years than in males aged $45-49$ years. The mortality rate was two times higher in females aged 50-54 years than in females aged $45-49$ years. The mortality rates were markedly increased in males aged $\geq 65$ compared with females of the same age group. The highest crude mortality rate occurred in males aged $\geq 85$, with 351 mortalities per 100,000 males 
A

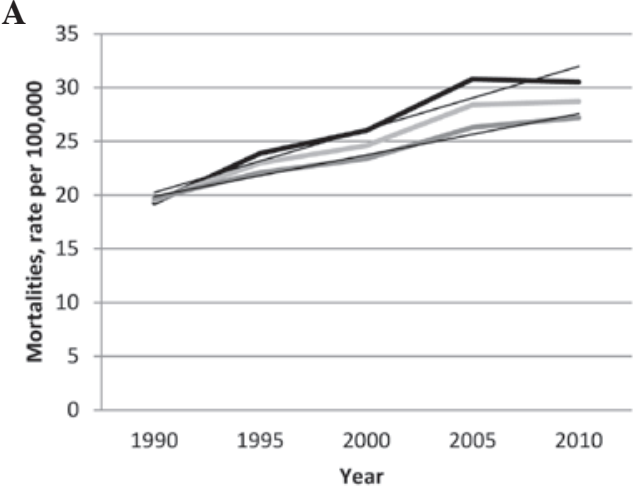

B

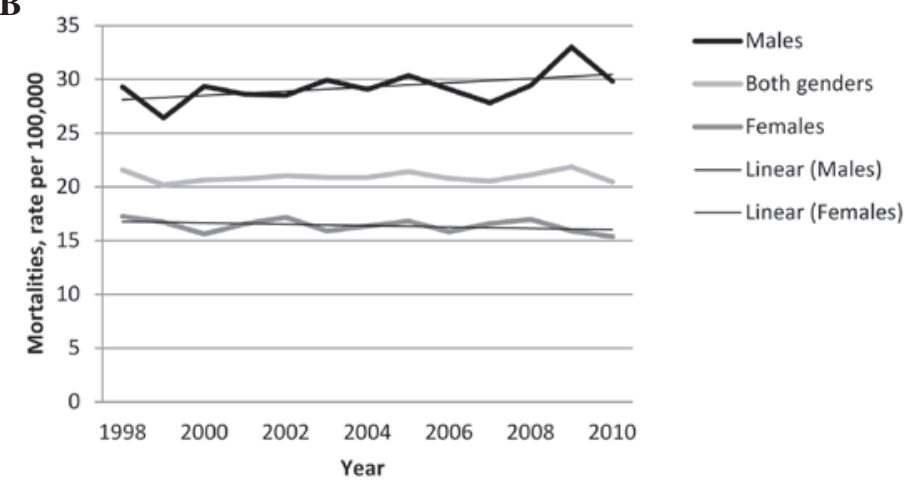

Figure 1. Age-standardised mortality rates of colorectal cancer for males, females and the two genders combined for all ages with linear trendlines between 1990 and 2010, based on (A) the Global Burden of Disease study data and (B) the European Detailed Mortality Database data for Lithuania.

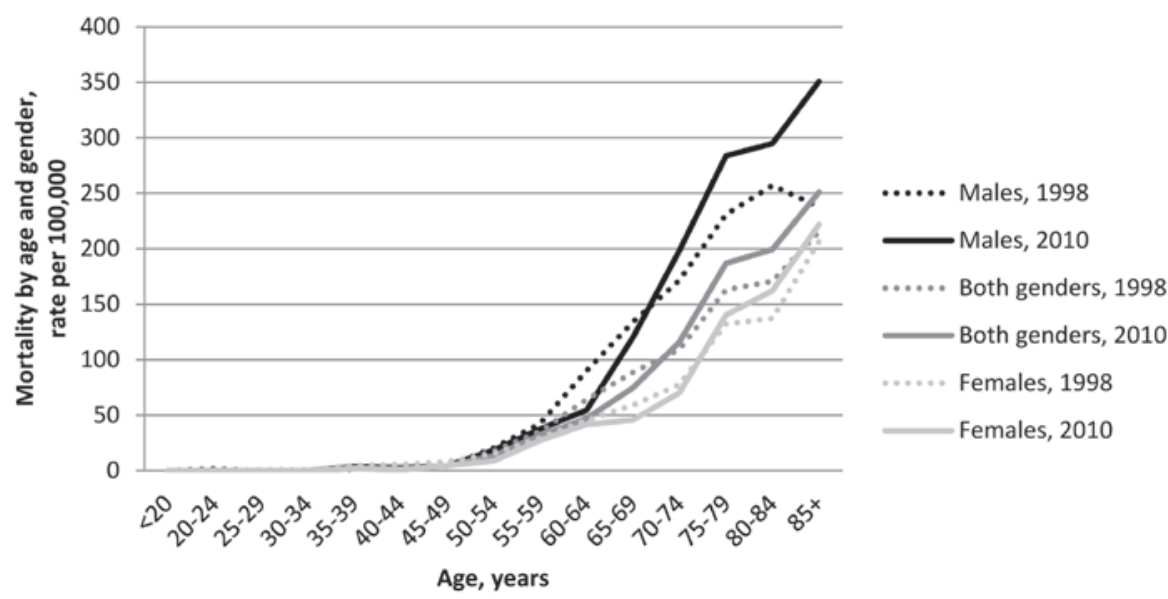

Figure 2. Distribution of the crude age-specific mortality rates from colorectal cancer by age and gender, and in the two genders combined in 1998 and 2010 in Lithuania.

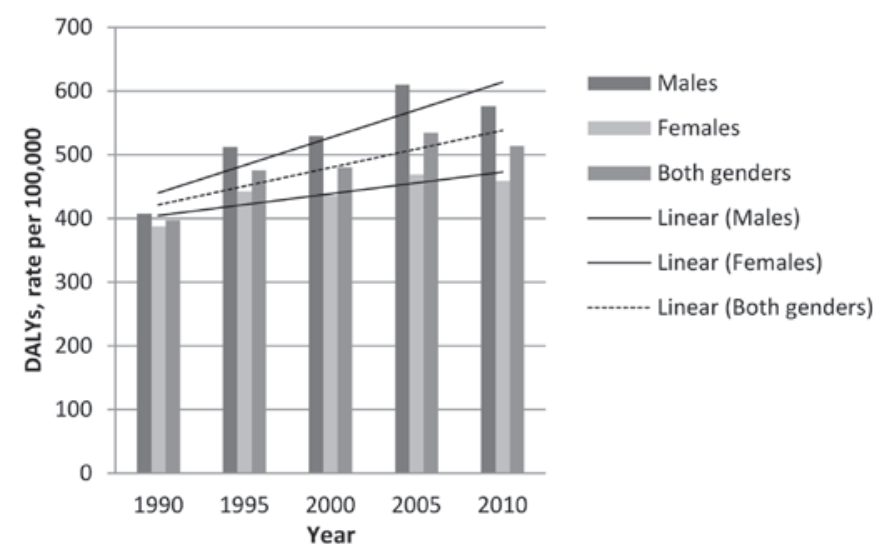

Figure 3. Number of DALYs caused by colorectal cancer between 1990 and 2000 in males, females and the two genders combined for all ages, with linear trendlines, in Lithuania. DALYs, disability-adjusted life years.

in 2010 . In the same year, the mortality rate in females aged $\geq 85$ was 222 mortalities per 100,000 females.

DALYs due to colorectal cancer. Fig. 3 reveals the number of DALYs per 100,000 individuals per year in Lithuania due to colorectal cancer between 1990 and 2010 in males, females and the two genders combined for all ages. The national burden of colorectal cancer was highest in 2005. In males, females and the two genders combined, the number of DALYs was decreased in 2010 compared with 2005. The burden of colorectal cancer was greater in males than females.

YLDs caused by colorectal cancer. The number of YLDs caused by colorectal cancer increased by $42.6 \%$ in the age-standardised population of 100,000 males, by $35.6 \%$ in the age-standardised female population and by $38.9 \%$ in the age-standardised overall population between 1990 and 2010. The number of YLDs increased between 11 and 19 years in males, between 12 and 18 years in females, and between 11 and 18.5 years overall in the two genders between 1990 and 2010.

Distribution of YLLs and YLDs due to colorectal cancer. In total, $97 \%$ of DALYs arising from colorectal cancer were from YLLs and 3\% of DALYs were from YLDs in 1990, 1995, 2000, 2005 and 2010. However, there is a slight nonsignificant trend towards a decrease in the number of YLLs and increase in the number of YLDs between 1990 and 2010. This trend is more evident in females than in males, as $3.0 \%$ of female DALYs were comprised by YLDs in 1990 and $3.9 \%$ were comprised by YLDs in 2010 . 


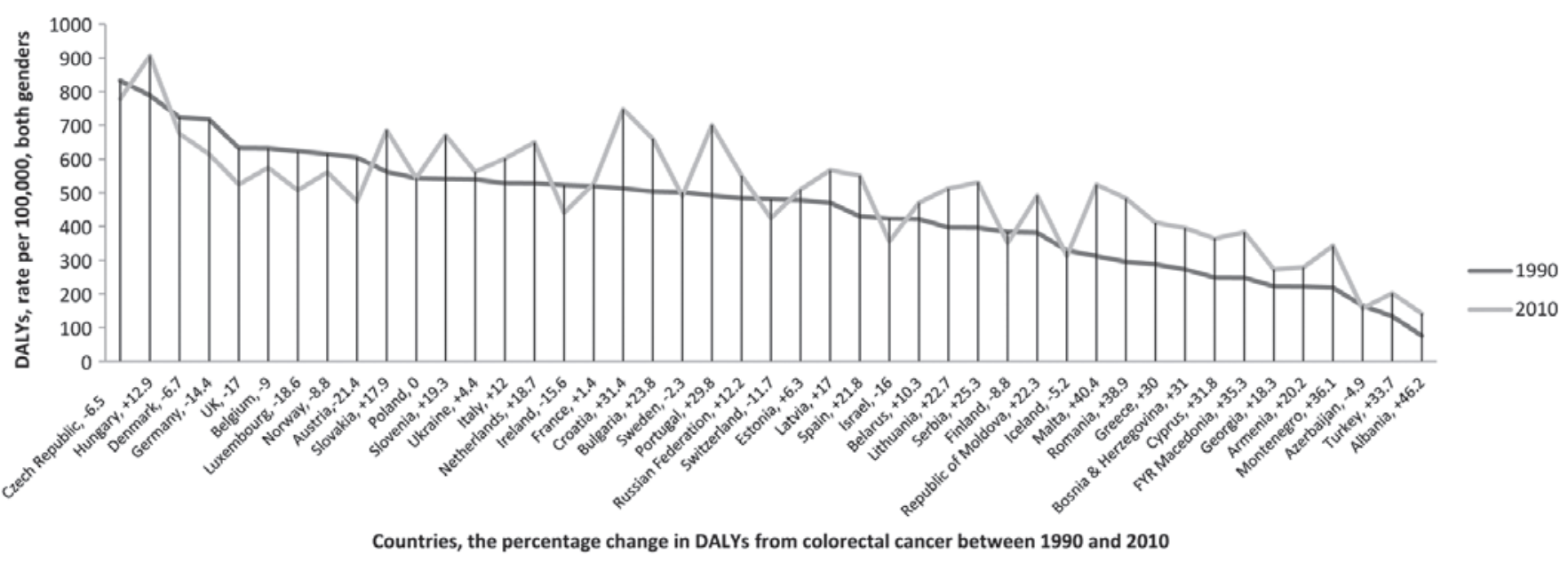

Figure 4. Age-standardised DALYs from colorectal cancer in both genders within 45 European nations in 1990 and 2010. DALY, disability-adjusted life years.

Ranking of DALYs due to colorectal cancer across 45 European nations. Lithuania was in the group of 14 European countries with the highest percentage increase in DALYs in males, demonstrating an increase of $29.3 \%$ (Fig. 4). This group of 14 countries includes Serbia, the Republic of Moldova, Bosnia and Herzegovina, Montenegro, Turkey, Cyprus, Portugal, Greece, Croatia, FYR Macedonia, Albania, Romania and Malta. Only 11 of the 45 European countries reduced the burden of colorectal cancer in males in terms of DALYs between 1990 and 2010. They are as follows, in descending order: Luxembourg; Finland; Israel; the UK; Austria; Switzerland; Azerbaijan; Ireland; Norway; Belgium; and Germany.

In females, Lithuania demonstrated one of the highest increases, with an increase of $15.5 \%$ in the burden of colorectal cancer from DALYs. In total, 17 countries reduced the burden of colorectal cancer in terms of DALYs between 1990 and 2010. These countries were as follows: Austria, which demonstrated the largest reduction of $32.4 \%$; Germany; Ireland; the UK; Luxembourg; Iceland; Belgium; the Czech Republic; Israel; Denmark; Switzerland; Belarus; Norway; Estonia; Sweden; Ukraine; and France.

The overall percentage increase in the national burden of colorectal cancer for males and females over 20 years in the Lithuanian population was $22.7 \%$.

Ranking of YLDs due to colorectal cancer across 45 European nations. In terms of male YLDs, Lithuania was ranked as 30th in 1990 and 29th in 2010 out of the 45 European countries. For female YLDs, Lithuania ranked slightly higher as 28th in 1990 and 27th in 2010. Over a 20-year period, every country, including Lithuania demonstrated an increase in YLDs. Lithuania was ranked as the 21 st country in males in terms of this increase, and as the 18th country in females. For males and females combined, Lithuania was ranked as the 20th country.

Ranking of YLDs across 12 European regions. The number of YLDs per 100,000 individuals per year in Lithuania increased by $22.4 \%$ between 1990 and 2010 . However, Lithuania was below the average of the Central and Eastern, Southern, Northern and Western regions of Europe in 2010. It was also below the average of Europe-45, EU-25, the original EU-15, the Nordic countries, the Baltic countries, the UK and the block of countries classified as democratic in 1990. In terms of YLLs per 100,000 individuals in 2010, Lithuania was above the average of the Central and Eastern European countries, Western Europe, EU-25, EU-15, the UK and the Baltic countries.

\section{Discussion}

The $30.6 \%$ increase in the overall crude incidence of colorectal cancer in Lithuania between 2001 and 2011 indicates that the incidence of colorectal cancer is likely to increase as the proportion of elderly individuals within the population of the Republic in Lithuania rises. Statistics taken in Lithuania reveal that the life expectancy at birth in Lithuania was 68.53 years in 1994, the lowest expectancy within the last 50 years, and 73.62 years in 2011 (13).

In the present study, the varying incidence of colorectal cancer between the regions of Lithuania was a notable finding for such a small country with a population size of 2.986 million in 2012 and an area of $62,302 \mathrm{~km}^{2}$ (13). The incidence varied twofold between certain counties and cities in Eastern and Western Lithuania. As the changing age structure of the population is similar in all Lithuanian regions, it was assumed that there are most likely numerous factors contributing to this discrepancy in the overall incidence rate of colorectal cancer in Lithuania at a particular time point or over time. In terms of the ranking of age-standardised incidence rates across European nations, the age-standardised incidence of colorectal cancer was revealed to be low in Lithuania compared with Western and Central European countries.

The mortality rates due to colon cancer varied considerably between the regions of Lithuania. Western Lithuania was revealed to be possibly the most challenging region in Lithuania as it demonstrates the lowest incidence of colorectal cancer as well as the highest mortality rates.

In general, it was found that the overall age-standardised mortality rate from colorectal cancer has increased in Lithuania since 1990. A number of points are noteworthy. First, the age-standardised mortality rate is higher in males than females. Secondly, there is a marked difference in the age-standardised mortality rates in males and females. Thirdly, different data 
sources report different values for the age-standardised mortality rates. This suggests that there are several areas for improvement in the retrieval of oncological data and statistics within Lithuania.

The assessment of DALYs presented a different interpretation to that provided by conventional mortality statistics, in which lung, prostate and stomach cancers are the three leading causes of mortality from malignant diseases in the Lithuanian male population (14). In the present study, it appeared that colorectal cancer was the third leading cause of the national burden of malignant disease in males and females. Furthermore, when the top 10 causes of the global and national burden of malignancies in Lithuania were compared and ranked by gender and YLDs, it was revealed that colorectal cancer is the second leading cause of disability (17.3\%) preceded by prostate cancer $(28.1 \%)$ in males. In females, colorectal cancer was ranked as the fourth highest contributor to disability, with 9.4\% of YLDs being due to this cancer.

In order to rank and define the performance of the Lithuanian health service provision for colorectal cancer, the geographic criteria were used for comparisons between European nation and regions, in addition to the GBD traditional metrics. When percentage change over the time was taken into account, it was revealed that Lithuania is one of a group of countries with a percentage increase in DALYs, and therefore YLLs, between 1990 and 2010. The percentage of DALYs rose by $22.7 \%$ between 1990 and 2010. This increase was more pronounced in males than females, with increases of 29.3 and $15.5 \%$, respectively. This suggests that the national burden of colorectal cancer in Lithuanian males was one of the highest in Europe. Only countries in the Southeast region of Europe, such as the Balkan Peninsula, demonstrated a higher percentage increase. Each of the five neighbouring countries of Lithuania, consisting of Poland, Latvia, Estonia, Belarus and the Russian Federation, demonstrated a larger decrease in DALYS than Lithuania. Estonia demonstrated a reduction DALYs in 2010, with a percentage decrease of $7.5 \%$ in females.

Lithuania demonstrates a relatively low number of YLDs due to colorectal cancer in comparison with other European countries and regions of Europe. This is the most discouraging finding of the present study, and suggests that novel therapeutic, surgical and radiological technologies for colorectal cancer management are not applied effectively in Lithuania (15). In addition, there is a substantial opportunity to improve colorectal cancer management through regionalisation, centralization and standardization. Finally, this finding indicates that a national colorectal cancer prevention programme is necessary in Lithuania.

This observational study demonstrates a number of important limitations. The use of multiple sources of data and estimates of the national burden of colorectal cancer is one limitation. As the present study assessed primary and secondary data, in addition to tertiary composite estimates, the appropriateness of the method applied for the assessment of the national burden of colorectal cancer may be disputable. In addition, it was impossible to evaluate the impact of additional risk factors and comorbid conditions on the mortalities due to colorectal cancer. Overall, the issues with the provision of colorectal cancer service in Lithuania may therefore be either underestimated or overestimated. The ranking of the 45 European nations may also be questionable due to the validity of the primary data sources of certain developing European countries remaining unknown.

In summary, the present study may provide a basis for the acceleration of the development of the Lithuanian colorectal cancer service and to implement long-term colorectal cancer prevention and control programmes.

\section{Acknowledgements}

The present study is a part of the 'Colorectal cancer in Lithuania: mortality, morbidity and quality of life study' project. The project is kindly supported by the National Research Council of Lithuania (grant no., LIG-01/2011). The authors also thank the International Agency for Research on Cancer, GLOBOCAN 2008 and the Global Burden of Diseases, Injuries, and Risk Factors Study 2013 team.

\section{References}

1. Murray CJL and Lopez AD (eds). The global burden of disease: a comprehensive assessment of mortality and disability from diseases, injuries and risk factors in 1990 and projected to 2020. Vol. 1. Harvard University Press, Cambridge, MA, 1996.

2. Wang H, Dwyer-Lindgren L, Lofgren KT, et al: Age-specific and gender-specific mortality in 187 countries, 1970-2010: a systematic analysis for the Global Burden of Disease Study 2010. Lancet 380: 2071-2094, 2012.

3. Murray CJ, Richards MA, Newton JN, Fenton KA, Anderson HR, Atkinson C, et al: UK health performance: findings of the Global Burden of Disease Study 2010. Lancet 381: 997-1020, 2013.

4. Highlights on health in Lithuania 2005. World Health Organization, Geneva, pp7-8, 2006.

5. Perlman S and Driver C; DALYs Working Group. Disability-adjusted life years (DALYs) in New York City. New York City Department of Health and Mental Hygiene Epi Data Brief 11, 2011.

6. Mathers CD, Vos T, Lopez AD, Salomon J and Ezzati M (eds): Planning a National Burden of Disease Study. In: National Burden of Disease Studies: A Practical Guide. Edition 2.0. World Health Organization, Geneva, pp16-23, 2001.

7. Jayatilleke N, Pashayan N and Powles JW: Burden of disease due to cancer in England and Wales. J Public Health (Oxf) 34: 287-295, 2012.

8. World Health Organization: Health statistics and information systems. Disease and injury country estimates: Burden of disease. http://www.who.int/healthinfo/global burden_disease/estimates_country/en/index.html. Accessed 21 September, 2013.

9. International Agency for Research on Cancer. World Health Organization. GLOBOCAN 2008: Cancer Incidence and Mortality Worldwide. http://www.iarc.fr/en/media-centre/ iarcnews/2010/globocan2008.php. Accessed 24 Sepember, 2013.

10. Ministry of Health of Lithuanian Republic: Institute of Hygiene Health Information Centre. http://sic.hi.lt/html/en/lhic.htm (In Lithuanian). Accessed 21 September, 2013.

11. World Health Organization: European Detailed Mortality Database. http://datanew.euro.who.int/DMDBPHP5/caparams. php. Accessed 21 September, 2013.

12. Institute for Health Metrics and Evaluation, University of Washington. Global Burden of Diseases, Injuries, and Risk Factors Study 2013. http://vizhub.healthdata.org/gbd-causepatterns/. Accessed 21 September, 2013

13. Statistics Lithuania. Official Statistics Portal. Life Expectancy. http://osp.stat.gov.lt/en/statistiniu-rodikliu-analize?portletForm Name $=$ visualization\&hash $=e 6729112-922 a-475 f-b 90 c-716$ dab8a 2f26. Accessed September 24, 2013.

14. Lithuanian National Cancer Institute. Lithuanian Cancer Registry. http://www.nvi.lt/index.php?-1413089819 (In Lithuanian). Accessed September 24, 2013.

15. Poškus E, Mikalauskas S, Jotautas V, Žeromskas P, Poškus T, Strupas K, et al: The pattern of colorectal cancer surgery in Lithuania in 2005: do results meet expectations? Medicina (Kaunas) 49: 124-131, 2013. 